\title{
Nkumi et nkumu. La sacralisation du pouvoir chez les Mongo (Zaïre)
}

Nkumi and nkumu. Making Power Sacred among the Mongo (Zaire)

Luc de Heusch

\section{Q OpenEdition}

\section{Journals}

Édition électronique

URL : http://journals.openedition.org/span/932

DOI : $10.4000 /$ span.932

ISSN : 2268-1558

Éditeur

École pratique des hautes études. Sciences humaines

\section{Édition imprimée}

Date de publication : 1 décembre 1990

Pagination : 169-185

ISSN : 0294-7080

\section{Référence électronique}

Luc de Heusch, « Nkumi et nkumu. La sacralisation du pouvoir chez les Mongo (Zaïre) », Systèmes de pensée en Afrique noire [En ligne], 10 | 1990, mis en ligne le 02 juillet 2013, consulté le 19 avril 2019. URL : http://journals.openedition.org/span/932 ; DOI : 10.4000/span.932 
NKUMI ET NKUMU

LA SACRALISATION DU POUVOIR CHEZ LES MONGO (ZAIRE)

par

Iuc de Heusch

\section{Nkumi}

Le pays mongo, dans la Cuvette centrale du Zaïre, est un cas de figure pour l'anthropologie politique. La région sud-ouest présente diverses formes de chefferies sacrées, totalement inconnues ailleurs. Le contraste est particulièrement frappant avec la région sud-est, où j'ai effectué une enquête durant deux ans, en 1953 et 1954. Ici, dans le nord du Kasaï, à la lisière de la grande forêt, les Tetela se caractérisent par une forte organisation lignagère où le pouvoir appartient aux aînés des divers segments; à l'échelle d'une tribu autonome, c'est l'aîné de la branche aînée qui exerce l'autorité sur l'ensemble du lignage maximal. L'aîné du lignage maximal aîné est le chef d'une tribu tout entière, mais les divers segments constitutifs de la société jouissent d'une grande autonomie. Ces aînés, tous appelés nkum' ekanga, ne sont investis d'aucun pouvoir rituel et leur prestige dépend essentiellement de leur générosité. Lors de leur investiture, les chefs des lignages maximaux, comme les aînés de segments inférieurs, acquièrent le droit de danser en public avec une peau de léopard sur le dos, au prix d'un vaste potlatch que j'ai

Systèmes de pensée en Afrique noire, 10, 1990 
décrit autrefois (de Heusch, 1954). Mais un proverbe affirme que le travail de chef (le devoir de générosité) ne finit jamais. Pour faire face à cette exigence, Kokolomami, le grand chef des Yenge, qui jouissait d'un prestige considérable dans la région, possédait un grand nombre de femmes. Chez les Tetela, le mariage fait du mari le débiteur permanent de son beau-père et de ses beaux-frères. Aussi bien est-ce à l'aide des aînés de son lignage que Kokolomami réussissait à remplir ses nombreuses obligations matrimoniales. Le chef de tribu n'est pas en droit d'exiger un tribut (sinon une part de tout gros gibier abattu) mais il bénéficie d'un certain nombre de dons perçus à titre d'hommage. Il reçoit un cadeau lorsqu'il est invité à se rendre en visite, lorsqu'il arrive chez son hôte ou s'en va. Tout chef de lignage maximal perçoit théoriquement "la chèvre de la tombe" lors des funérailles; il est obligatoirement gratifié d'un don lorsqu'un léopard est abattu par un chasseur dépendant de lui, etc. Mais, de l'avis général, les chefs se dépensent pour les leurs et le principe de la générosité est au premier plan de la définition du pouvoir. Celui-ci se manifeste principalement dans le domaine judiciaire; c'est un conseil d'aînés qui tranche les différends locaux; seuls les cas graves sont évoqués devant le tribunal constitué par l'ensemble des chefs de lignage.

Les chefs de lignage maximaux sont entourés chez les Yenge d'un corps de guerriers, les ahuka, qui exercent aussi la fonction de messagers. Leur comportement quelque peu matamoresque effraie les enfants. Ils participent aux enterrements et étaient jadis les exécuteurs des hautes oeuvres. Ils ont mauvaise réputation. Ce petit groupe remuant s'agite autour des étrangers de marque lors de leur arrivée au village: le visage barbouillé de noir, ils brandissent une lance menaçante. Ce comportement amuse l'assistance qui n'est pas dupe du jeu. Cette petite troupe est mixte: elle est composée des descendants, hommes ou femmes, de guerriers qui peuvent s'enorgueillir d'avoir tué un ennemi au combat. Ces guerriers saluent aussi bruyamment le chef de lignage le jour de son investiture lorsqu'il surgit de la forêt, accompagné de ses femmes et de ses proches 
parents, tacheté de blanc pour imiter le pelage de l'animal. Dans ce cortège, les uns portent la peau du léopard sur le dos, les autres exhibent seulement un collier de dents de léopard. Cette assimilation du chef et des siens à l'animal le plus fort et le plus redoutable de la forêt est $d$ 'ordre métaphorique. Le rite ne confère en aucune façon au chef confirmé dans sa fonction le pouvoir de se transformer en fauve, ce qui est notamment le cas du roi sacré des Kuba lorsqu'il désire se venger d'une offense. Mais la métaphore est suffisamment forte pour qu'elle interdise au chef et aux membres de son segment de lignage de consommer la chair de cet animal.

Le pouvoir est investi dans l'ordre familial chez les Tetela; il n'est que l'extension au niveau du lignage (plus ou moins étendu) du pouvoir du père. Il n'implique aucune mainmise sur les hommes et les produits de leur travail. Il se monnaie dans une économie de potlatch où il importe de se dépenser sans arrêt. Tout est affaire de prestige ici. Il existe chez les Yenge des chefs de lignage maximaux fort peu considérés mais l'autorité de Kokolomami, l'aîné de tous les Yenge, dont la devise proclamait: "le tonnerre du ciel, tous les hommes l'entendent", était considérable.

Maître théorique de la terre (owandji wa nkete), le chef de tribu, pas plus que le chef de lignage, ne détient de pouvoir magicoreligieux. Seuls les devins-guérisseurs, regroupés dans la même association que les forgerons, ont la faculté d'entrer en rapport avec les esprits errants de la nature (edimu) qui constituent l'une des sources majeures du malheur et de l'infortune.

La configuration politique est fort différente dans la région forestière avoisinante, dans les tribus hamba, qui sont étroitement apparentées aux Tetela au sein d'une même généalogie. Hamba et Tetela de la savane affirment tous descendre du même ancêtre, Ankutshu a Membele. Dans la forêt les grands lignages se sont émiettés et l'on a vu surgir une institution originale, la société fermée des nkum'okuna (maîtres de la forêt). Celle-ci s'oppose de manière évidente au désordre des guerriers ahuka et tend à vider le pouvoir de toute connotation violente. Le symbole par excellence du nkumi c'est 
la cloche elundja, symbole de paix. Lorsqu'un nkumi bat l'elundja, la querelle s'apaise. Cette association fermée qui s'est imposée au-delà de l'horizon tribal, comporte de multiples grades, variables d'une région à l'autre. Ils s'obtiennent grâce à des paiements distribués aux membres. Lorsque chez les Djumbusangaa ou les Djembu, le candidat sollicite le droit de battre l'elundja, on le met en présence d'un choix décisif entre les armes et la cloche. On adjure alors le candidat de se soumettre aux règles morales et de se montrer généreux envers les autres nkumi d'abord, mais aussi envers tous les hommes. On voit prendre corps ici une certaine image du nkumi comme big-man: il se promène avec une espèce de gibecière à l'épaule ou un panier de femme sur le dos, dans lesquels se trouve symboliquement de la nourriture ou des richesses qu'il est censé distribuer. L'entrée dans l'association et l'acquisition des divers grades ultérieurs s'obtient elle-même par la générosité en distribuant des monnaies de cuivre aux autres nkumi.

Le nkumi se déplace avec une lance de prestige (en fer rehaussé d'un fil de cuivre) qui s'oppose explicitement à la lance de guerre: lorsque le premier emblème est planté dans le sol, il met fin aux querelles et aux disputes. L'assemblée des nkumi constitue le tribunal du village ou d'une région. Les nkumi partagent à cet égard le pouvoir avec les chefs de lignage qui font partie généralement de l'association. Ceux-ci y occupent le plus souvent des fonctions éminentes. Chez les Djembu ils contrôlent les rites d'initiation, ils ont le dernier mot dans les palabres.

Cette organisation supra-familiale crée un réseau de solidarité entre les villages et les tribus: tout nkumi en voyage bénéficie en effet de l'hospitalité de ses pairs et participe pleinement aux réunions de ses hôtes avec des droits égaux. Les nkumi se réservent un certain nombre d'animaux, dont la liste varie d'une région à l'autre. Ils interviennent parfois auprès des ancêtres en cas d'insuccès de la chasse au titre de "maîtres de la forêt", mais ils n'exécutent aucun rituel proprement religieux. Ils forment plutôt un club récréatif où l'on prend plaisir à chanter et à danser en imitant le comportement des animaux. 
Ce pouvoir acquis par des dons s'oppose symboliquement à l'ordre familial et lignager. Au moment où il reçoit la cloche elundja, dont le son appelle la concorde, le récipiendaire rompt un interdit significatif : chez les Djumbusanga comme chez les Djembu, il évoque le sexe de son père et de sa mère. Le nkumi, dit-on, doit être au-dessus de la honte. Mais le nkumi est le défenseur patenté de la morale et du droit et à ce titre il n'est plus solidaire de sa famille au cas où un proche parent serait impliqué dans un conflit. Il doit juger les siens avec la même impartialité que les membres des autres lignages.

La société intertribale des nkumi exerce son pouvoir sur l'ensemble du territoire. C'est pourquoi chez les Yenge, où l'institution commençait à pénétrer il y a une trentaine d'années, le nouveau nkumi "achète" les forêts et les plaines. Chez les Udjangi ces paiements se multiplient de manière fantasmatique: le candidat "achète" tour à tour le droit de franchir un arbre abattu, d'écraser les termitières, etc. Tout se passe comme si, au niveau du langage au moins, les nkumi tentaient d'empiéter symboliquement sur le pouvoir des chefs de lignages maximaux, maîtres de la terre. Nkumi signifie d'ailleurs "chef". Mais leur domaine est plus spécialement la forêt, réserve inépuisable de gibier.

Ce pouvoir ne cesse d'être associé au léopard. L'acquisition d'un collier de dents de fauve constitue partout l'un des grades majeurs. Mais ce n'est plus la violence du léopard qui est exaltée ici: au contraire, dans les rites, les nkumi imitent la démarche réservée et prudente de cet animal. Ils créent ainsi un espace de paix qui contraste avec l'agitation des guerriers qui, dans la savane, se démènent bruyamment autour des grands chefs de lignages, métaphoriquement assimilés à de dangereux fauves. Il est significatif que l'institution des nkumi ait provoqué la disparition du corps constitué des guerriers héréditaires chez les Hamba de la forêt. Lorsqu'ils se réunissent à quelque distance du village, dans une enceinte interdite, protégée par un mur de feuillage, les nkumi doivent déposer sur le sol leurs couteaux, affirmant ainsi leur vocation pacifique. Mais ce lieu de paix est aussi celui où, théoriquement, les maîtres de la forêt exercent 
collectivement leur droit de vie et de mort. Chez les Djembu on peut y voir notamment une petite potence miniature qui rappelle que les criminels étaient pendus jadis en vertu d'un jugement rendu par la confrérie.

La société des maîtres de la forêt investit donc totalement l'espace du pouvoir dévolu chez les Tetela de la savane aux seuls chefs de lignage. Il s'agit là d'une transformation récente et toutes les traditions des Hamba disent clairement que le berceau de l'institution se trouve chez une population septentrionale, les Ankuthsu. Cette révolution politique pacifique n'a pas altéré profondément le sens même de l'autorité: c'est toujours par la générosité que le nkum'okunda (maître de la forêt) comme le nkum'ekanga (chef de lignage) maintiennent leur prestige et acquièrent ainsi une relative autorité. A l'inverse du second, le premier est impuissant à imposer le contrôle de l'ordre à titre individuel: il ne peut agir qu'au sein d'une confrérie, qui tire une partie de son autorité du secret dont s'entourent les rites d'initiation. Ceux-ci sont variables d'une région à l'autre et ne s'accompagnent d'aucun enseignement mythique. La sphère des maîtres de la forêt est indépendante du domaine magico-religieux, comme l'est la définition du pouvoir dans l'ordre lignager.

\section{Nkumu}

\section{A. Ntomba}

Tournons-nous à présent vers les Mongo du sud-ouest. Nous nous trouvons en présence d'une véritable révolution idéologique; le passage du nkumi au nkumu se marque en effet par l'émergence du pouvoir sacré. Celui-ci est désigné du terme ekopo, qui désigne la peau de léopard (Müller, 1955: 3 et Sulzmann, 1959: 393). Un article datant de 1930 (publié en 1939) nous décrit cette étrange figure chez les Ntomba méridionaux (Stas, 1939: 109-123). Stas se limite à la description des nkumu de haut rang de la région de Bikoro (Müller, 1955: 3) 
Le nkumu cumule les fonctions de juge et de magicien-guérisseur, toujours soigneusement distinctes chez les Hamba-Tetela. C'est par son intermédiaire que les hommes communiquent avec les ancêtres et les esprits de la nature (elima). A ce titre, il préside aux destinées du groupe. C'est notamment lui qui désignera l'emplacement des nouveaux villages. Il dirige les cérémonies funéraires au cours desquelles des libations de vin de palme sont répandues sur les tombes. C'est là aussi que le nkumu intervient auprès des ancêtres lorsqu'un fléau s'est abattu sur le territoire. Exorciste, il a le pouvoir de chasser l'esprit elima qui empêche une femme d'accoucher, ou de l'apaiser lorsqu'il entrave le succès de la chasse. Mais il peut aussi paralyser cette activité par ses sortilèges si la part du gibier abattu qui lui est dû au titre de tribut ne lui est pas apportée.

Ces pouvoirs considérables s'acquièrent par des dépenses ostentatoires suivies d'une initiation qui porte la marque de la rupture. Le nkumu est véritablement un big-man investi d'un pouvoir rituel. Le candidat, le corps barbouillé de noir de charbon, commence par rendre visite à l'ensemble de sa parenté pour rassembler les nombreuses monnaies de cuivre qu'il devra distribuer pour acquérir la dignité de nkumu: celle-ci s'acquiert donc par une démonstration de générosité, comme la qualité de nkumi chez les Hamba. Mais la dignité de nkumu diffère radicalement de celle-ci. Alors que les nkumi agissent toujours collectivement, le nkumu est seul face à son groupe; en outre, et la différence est de taille, les nkumi hamba (pas plus que les chefs de lignage tetela) ne se voient pas attribué de pouvoir rituel.

Or, le nkumu, quant à lui, fait véritablement figure de chef sacré. $\mathrm{Au}$ début de la cérémonie d'investiture, il se rend la nuit dans le cimetière où reposent ses ancêtres, accompagné de sa parenté et des gens importants du village, pour obtenir l'agrément des défunts. Les épouses du candidat le débarrassent de la couleur noire qui le recouvrait et le fardent en rouge. Il demeure enfermé dans sa maison durant un mois. L'accès en est interdit au moyen d'une barrière et le nkumu doit acheter le droit de sortir de chez lui. Au terme de cette période de réclusion, tous les chefs de famille sont invités à 
recevoir leur part du prix de l'investiture. Le candidat dépense "une fortune" (Stas, 1939: 115). Le nouveau nkumu acquiert dès lors le droit de prélever des tributs sur la chasse et la récolte.

Etrange marché à vrai dire. Il ne s'agit évidemment pas d'un acte de pure réciprocité car le potlatch, fût-il exorbitant, ne compense évidemment pas la créance perpétuelle que le nkumu détient sur le groupe. En fait, le nouveau chef est censé apporter par la voie des rites d'immenses bénéfices à la société. Et ce pouvoir est d'un ordre particulier, il ne prend pas sa source dans l'ordre familial. Au contraire, il lui est extérieur, il est d'ordre transcendant. Le premier des nkumu, Mungu-Mpembe, était une créature d'origine céleste, munie d'une petite queue. Il relève donc de l'ordre supra et infraculturel (il ressemble à un animal). L'on ne connaît rien de sa famille ni de sa patrie (Stas, 1939: 118-121). C'est un être solitaire et déraciné qui s'est imposé par sa magie. Cet étranger prit possession de la terre de Ntomba par un acte de souveraineté magique. Il planta son pied dans le rocher où son empreinte s'inscrivit comme s'il s'enfonçait dans de la terre molle. Il fut aperçu quelques temps après par une femme qui prit peur à la vue de ce "géant". Mais Mungu-Mpembe la retint et se présenta comme un seigneur doté d'une grande puissance. La femme s'en retourna annoncer la nouvelle à son mari. Celui-ci refusa d'accueillir l'étranger mais son frère y consentit. Mungu-Mpembe lui fit présent de sept cents barres de cuivre. Mais les hommes n'étaient pas disposés à recevoir cet inconnu et sa suite. Celui-ci accomplit alors un prodige: en un instant une petite termitière se mit à grandir pour at teindre la hauteur d'un arbre. Les autochtones furent impressionnés par cet exploit et se montrèrent plus accommodants .

Ce court mythe est fort instructif. Il rend compte de la nature ambiguë du pouvoir nouveau, l'ekopo: introduit par un potlatch, il s'impose par une démonstration de puissance magique. Le mythe rappelle complaisamment l'efficacité des médecines et des sortilèges de Mungu-Mpembe, héros culturel qui apprit aux Ntomba l'art de fabriquer des pots de cuisine et des pirogues. Ce portrait quasi frazérien d'un chef qui s'impose par l'exercice de sa magie ne retrace 
évidemment pas un processus historique; il rend compte de la nature rituelle d'un pouvoir sacré venu d'ailleurs. Ce big-man généreux et protecteur est censé avoir réuni autour de sa personne un nombre considérable d'hommes; il distribua l'ekopo à d'autres, et c'est ainsi que les nkumu se multiplièrent. Il prit le titre de Yeli et percevait des droits importants lors de l'investiture d'un nouveau titre. Tous les villages furent ainsi dotés d'un nkumu. Au village principal de Mpasha, l'on compte une douzaine de successeurs de Mungu. Celui qui régna entre 1850 et 1880 fit régner la terreur.

Un second article datant de 1944 apporte des précisions complémentaires sur la "chefferie rituelle" des mêmes Ntomba (Tumba), institution aujourd'hui disparue. Cette étude, due à H.D. Brown, porte plus spécialement sur l'un des sous-groupes ntomba de Bikoro, les Bonginda (cf. Müller, 1955: 3).

La société ntomba se compose de familles étendues placées sous l'autorité de patriarches, maîtres du sol. L'institution politique de l'ekopo rompt avec ce système patriarcal car le nkumu, choisi par un village ou un quartier, est toujours un étranger riche, capable d'effectuer les nombreux paiements liés à l'acquisition de sa charge. L'insigne principal de sa fonction est un chapeau de vannerie, orné de deux grands disques de cuivre. Curieusement, lorsque ce couvrechef est présenté au nouveau nkumu, l'on assiste à un véritable marchandage. Le candidat commence par offrir un nombre dérisoire de barres de cuivre; on le presse alors d'en donner davantage et la scène se répète jusqu'à ce qu'une centaine d'unités aient été livrées. Durant la retraite d'un mois, la femme rituelle du nouveau nkumu, la nsono, est accapareuse de nourriture. Assise sur le siège du nkumu, silencieusement, elle dépouille de leur charge tous ceux qui passent devant elle avec des vivres. Font exception à cette règle les membres du groupe qui a appelé le nkumu. Désigné du terme molembe, ce groupe fera figure collectivement de "mari" du nkumu. Celui-ci et les membres de sa famille doivent partager avec les membres du molembe le léopard, l'éléphant et d'autres animaux abattus à la chasse. Mais les membres du molembe font de même vis-à-vis du nkumu et des siens. Lorsque 
ce dernier sort de sa retraite, à la fin des rites d'investiture, il doit se dépenser largement en honorant par un don tous ceux qui viennent lui rendre hommage. Le poids économique de la charge est donc considérable. Comme le chef de lignage tetela, le nkumu ntomba accède à sa dignité par un véritable potlatch. A cet égard les deux institutions sont structurellement apparentées, bien que la seconde se sépare radicalement de la première par la nature même de la fonction.

Un trait caractéristique apparente le nkumu aux chefs sacrés frazériens: il ne peut mourir de mort naturelle. Lorsque sa fin approche, les pygmées twa qui sont à son service viennent l'étrangler. De nombreux esclaves sont tués et mangés à l'occasion de ses funérailles.

\section{B. Ekonda}

La même tradition relative à la mort rituelle du nkumu a été rapportée chez les Ekonda (Van der Kerken, 1944:640). Ici le nkumu est l'intermédiaire entre le village et un génie local (elima) responsable de la fécondité des femmes et du succès à la chasse (Müller, 1955: 7). Cet esprit est essentiellement bienveillant, mais il est susceptible de se courroucer; un feu brûle en son honneur dans la maison du nkumu. Aux yeux des mêmes Ekonda, le nkumu est dangereux pour la communauté durant la retraite qui précède l'investiture car l'esprit protecteur elima n'est pas encore fixé dans la maison rituelle qui ne sera construite qu'à l'occasion de l'installation. Après son investiture, un contact permanent s'établit avec l'elima protecteur par l'intermédiaire de l'anneau de fer que le nkumu porte au poignet gauche. Tout contact avec sa personne est prohibé. Le nkumu ne prenait jamais part à la guerre. Durant les combats, il demeurait assis sur sa chaise. La fonction d'intermédiaire entre le village et l'elima place le nkumu "dans une situation tout à fait exceptionnelle" (Müller, 1957: 281-283).

Chez les Ekonda, le nkumu perçoit un tribut de chasse. L'aigle et le léopard lui appartiennent et on lui remet obligatoirement une part d'un certain nombre d'animaux de grande taille. Il exerce aussi 
des droits sur la personne des pygmées. Ceux-ci "appartiennent"au titre de dépendants à des chefs de famille particuliers, mais le nkumu a le droit d'exiger des corvées de tous les Twa du village où il exerce son autorité morale. Enfin, il est investi d'une autorité judiciaire (Müller, 1955: 12): il préside le tribunal villageois (Müller, 1957).

\section{Bolia}

L'ekopo est une forme embryonnaire de pouvoir royal. Le mythe d'origine des Ntomba méridionaux relate qu'Ilanga, la soeur du premier nkumu Mungu Mpembe, régna sur les Bolia matrilinéaires. Or ceux-ci développèrent un véritable appareil d'Etat autour de la figure d'un chef sacré qui porte le nom même d'llanga. Vansina estime que cette formation politique se serait développée entre le XIVe et le XVIe (Vansina, 1965: 80). Le royaume comprenait quatre provinces, gouvernées par des chefs héréditaires, issus du même ancêtre féminin Mpata. Ces quatre lignages assumaient la royauté à tour de rôle (Sulzmann, 1959).

E. Sulzmann ne doute pas que l'ekopo est chez les Bolia le fondement d'un pouvoir politique appuyé sur le système religieux (idem, p.394). Le prétendant au trône qui surgit dans le lignage qualifié doit affronter un certain nombre d'épreuves magiques pour être désigné. Les ancêtres, qui lui apparaissent en rêve, lui confèrent le don de prédire les prochains événements. Ils l'entraînent aussi auprès du souverain des esprits de la nature, Mbomb'Ipoku. Pour arriver à sa demeure souterraine le candidat doit marcher sur un long tronc d'arbre glissant au risque de tomber dans l'abîme (idem, p.404).. Le pacte conclu avec ce grand génie est l'élément essentiel du pouvoir sacré (Gilliard, 1925: 229). Il situe celui-ci d'emblée dans la zone inquiétante de la sorcellerie car le candidat doit livrer au maître du monde surnaturel un certain nombre de victimes humaines choisies dans sa parenté. "Si j'avais encore mon père, ma mère, et mes frères et mes soeurs, serais-je encore en vie" déclare étrangement le chef suprême des Bolia à Gilliard. Durant son investiture, le candidat rend visite à divers génies au cours d'un long périple. L'un de ceux-ci, à qui 
il doit livrer une femme, lui procure le pouvoir de contrôler la pluie. Pour prouver la réalité de ces contacts avec le monde des esprits de la nature, le candidat doit pouvoir soulever une formidable tempête. Le jour de son intronisation, il communique directement avec la nature: lorsqu'il reçoit le pot rituel contenant du kaolin, symbole de souverajneté, tous les animaux de la forêt le saluent de leur chant ou de leur cri (Gilliard, 1925: 230-234). E. Sulzmann présente une version légèrement différente: à la fin des épreuves préliminaires, le candidat appelle les animaux nocturnes qui lui répondent, attestant ainsi la bénédiction de Mbomb'Ipoku. C'est lorsque ces voix se font entendre que les autres prétendants au pouvoir abandonnent la partie. Aussitôt après la réponse des animaux, le candidat perd connaissance. Lorsqu'il revient à lui, il personnifie désormais Iyebelo i Mbomba, l'ancêtre de la dynastie, le plus jeune des triplés nés de la mère originelle Mpata qui épousa son frère utérin (Sulzmann, 1959: $406 \& 396$ ). On ne pourrait dire plus clairement que le pouvoir royal s'enracine dans un univers naturel, marqué notamment par l'inceste et une fécondité naturelle excessive.

Cette sphère du pouvoir magique, je viens de le souligner, est aussi celle de la sorcellerie. Je développerai à présent cet aspect des choses, sans doute le plus surprenant. Le rôle central de Mbomb'Ipoku dans l'acquisition du pouvoir sacré est précisé par Nestor Van Everbroeck (1961). Ces indications précieuses établissent clairement que tout pouvoir se construit, chez les Bolia, sur une transgression.

Mbomb'Ipoku, le chef des esprits de la nature elima, ne fraie qu'avec les grands chefs. Ceux-ci n'entrent en contact avec lui qu'une seule fois dans leur vie, au moment de leur investiture. Ils entretiennent ensuite des rappors plus familiers avec les elima subalternes qui sont leurs épouses. Tout homme est susceptible de communiquer avec un elima à condition de lui être présenté par un ancêtre. Le prix de cette rencontre est toujours une vie humaine. L'ancêtre apparaît en songe à l'un de ses descendants et lui fait part de son désir de l'initier au pouvoir rituel iloki en l'avertissant qu'il devra offrir préalablement la vie d'un proche (neveu utérin, soeur, 
mère, enfant, épouse) à un esprit elima (Van Everbroeck, 1961: 77 et 87). Le forfait est accompli par l'ancêtre en personne, assisté de l'ombre (esisa) de l'intéressé.

Cette initiation à la sorcellerie de clan (iloki i bokundi) confère de dangereux pouvoirs destructeurs mais son bénéficiaire ne pratiquera pas nécessairement cet art redoutable. En tout état de cause, ce n'est qu'après avoir bénéficié de cette connaissance que l'on peut ambitionner d'accéder à une charge politique, forme spécialisée de la sorcellerie. Van Everbroeck distingue deux formes de l'iloki: l'iloki i loboko, le pouvoir sur le sol, et l'iloki y ekopo, le pouvoir politique royal. Il est indispensable d'acquérir auprès d'un esprit elima, moyennant une vie humaine, le pouvoir sur la terre (nkumu e loboko). Cet échange confère la faculté de "connaître tout ce qui se passe aussi bien dans le monde visible qu'invisible", sur l'étendue du domaine dont il est propriêtaire. L'esprit sollicité se montre bienveillant: il explique au nouveau chef de terre comment il doit s'y prendre pour assurer la prospérité générale, comment combattre l'action criminelle des sorciers. Il lui confère aussi le pouvoir de maudire le sol et ses habitants pour asseoir son autorité. L'iloki y ekopo désigne l'essence du pouvoir politique supérieur (littéralement la "sorcellerie" de l'ekopo). L'aïeul initiateur transporte le candidat-chef dans les ruines de son ancienne résidence. Il convoque tous les esprits du village qui, moyennant la promesse d'une vie humaine, leur confèrent à tous deux la force de se rendre auprès de leur chef Mbomb'Ipoku. A celui-ci le candidat-chef devra livrer plusieurs victimes, choisies dans sa parenté, pour être investi "du pouvoir sur les hommes, les animaux et les poissons de sa future chefferie" (Van Everbroeck 1961: 92-95).

On aimerait évidemment en savoir davantage sur les rapports entre les chefs du sol et les détenteurs de l'ekopo, cette puissance supérieure venue d'ailleurs, transmise à l'origine par un géant venu du ciel (Ntomba) ou par Mbom'Ipoku "le seigneur de l'abîme" (Bolia).

Cette brève incursion chez les Mongo du sud-ouest montre comment la figure du big-man, est susceptible d'incorporer des valeurs qui sont fondamentalement étrangères aux Mongo du sud-est (Tetela-Hamba) 
pour donner naissance à une conception radicalement différente du pouvoir. Qu'elle soit d'essence purement magique ou qu'elle s'enracine au moins partiellement dans la sorcellerie, l'autorité associée au concept ekopo se situe à un point de rupture. L'ordre politique des chefs de lignage chez les Tetela, ou l'association intertribale des maîtres de la forêt chez les Hamba, maintiennent fermement le pouvoir à l'intérieur de l'ordre social: quels que soient les droits matériels secondaires dont il bénéficie, le détenteur de l'autorité (à titre individuel ou collectif) est théoriquement le dé-biteur du groupe qui assure un débouché à sa vanité. En revanche lorsque, chez les Ntomba, un homme ambitieux dépense avec ostentation pour devenir nkumu, il acquiert des droits considérables; il perçoit un tribut en vertu des pouvoirs surnaturels qu'il se voit conféré avec un mélange ambigu de crainte et d'admiration. C'est par le détour de la magie que le bigman se trouve au fondement de la royauté bolia.

On ne s'étonnera pas dès lors de voir Vansina attribuer une origine mongo à la royauté kuba. Rappelons la dernière position de cet auteur. De nombreux rapprochements permettent d'affirmer que les ancêtres des Kuba étaient apparentés aux Mongo du sud-ouest. En particulier le concept d'autorité sacrée ekopo doit être rapproché du principe fondamental de la royauté kuba: il ne dérive pas de la seniorité dans l'ordre familial mais s'enracine dans le contrôle d'un territoire. L'ekopo des Bolia transcende l'ordre familial dans la mesure où il se réfère à des esprits du sol.

Le terme ekopo apparaît sous des formes diverses dans la tradition orale kuba et le complexe de la royauté sacrée pourrait fort bien dériver du pouvoir mystique que certains chefs territoriaux (kum) acquièrent en pays mongo auprès des esprits de la nature, dont dépend leur légitimité. (Vansina, 1978: 97). Le roi kuba, assimilé à un esprit de la nature, maître absolu de la fécondité et de la fertilité, est à la fois magicien et sorcier. Il ne livre aucun membre de sa famille à une divinité cannibale mais il s'unit incestueusement (et en secret) à une soeur, puis épouse une petite nièce de son lignage au moment de son investiture. Le roi, tel un sorcier, a la faculté de se transformer 
en léopard pour se venger de ses ennemis. Tel un sorcier, il s'identifie à l'ordure (Vansina, 1964: 100, $103 \& 110$ ).

Au-delà de ces liens historiques directs, il existe entre la problématique de la chefferie sacrée telle que la posent les Ntomba et la geste de fondation du royaume luba du Zairre méridional des liens structuraux. On se souviendra que le mythe d'origine de l'ekopo attribue à un étranger, d'origine céleste, Mongu Mpembe, l'introduction des mystérieux pouvoirs de l'ekopo. C'est encore un étranger, un chasseur cette fois, qui introduit le principe de la royauté sacrée (bulopwe) chez les Luba. (de Heusch, 1972, chap. I).

Prince du monde d'en haut, homme de belle prestance, séducteur, Mbidi Kiluwe octroie généreusement aux autochtones qui l'accueille le gibier, comme Mongu Mpembu le géant leur distribue des monnaies de cuivre. Le chasseur et le big-man se trouve occuper la même position de donneur par rapport à un groupe d'accueil auquel ils apportent le principe magique de la royauté .

Dans les deux cas le pouvoir sacré fait irruption de l'extérieur, sans violence. Etre déterritorialisé, le chef venu d'ailleurs impose un nouveau type de relation politique par la voie retorse de la séduction. On mesure dès lors la valeur exemplaire de l'opposition que nous avons tracée d'entrée de jeu entre les Mongo du sud-est, qui ignorent toute espèce de sacralisation du pouvoir, et les Mongo du sud-ouest, où celle-ci fait irruption au sein d'un système de pensée également fondé sur la prééminence du big-man. L'Afrique centrale offre un modèle de transition entre deux formes de pouvoir qui se trouvent dans un rapport d'incompatibilité en Océanie. Il semble bien en effet exister là-bas un hiatus insurmontable entre les sociétés mélanésiennes à big-men et les formes polynésiennes de la royauté sacrées. Pierre Clastres a mis l'accent sur cette opposition dans son introduction à l'oeuvre de Marshall Sahlins. Il a bien montré qu'il existe une opposition radicale entre la position du big-man mélanésien, qui se trouve perpétuellement en dette vis-à-vis du groupe qui maintient en laisse son ambition effrénée, et celle du chef polynésien, qui a la capacité d'imposer le tribut (Clastres, 1976). 
Si en Afrique Centrale, le big man réussit parfois à opérer le renversement de la dette, en imposant le tribut à ceux envers lesquels il s'est d'abord montré généreux, c'est en vertu d'une nouvelle idéologie, celle-là même qui se trouve au fondement de la royauté sacrée: l'efficace du pouvoir se trouve dans l'exercice ambigu de la puissance magique. Sur ce point fondamental, Frazer ne s'était pas trompé. Il n'a pas aperçu cependant que la royauté magique se situe à un tournant décisif des transformations structurales du pouvoir. Le big-man généreux ne peut transformer son prestige en autorité qu'à la condition de prolonger dans le monde imaginaire sa fonction de donateur. Il doit faire alliance avec le monde des esprits pour que sa générosité apparente devienne le ciment de la royauté. Ce nouveau consensus, fondé sur une feinte suprême, n'a cessé d'être l'idéologie du pouvoir sacré ou divin dans les sociétés historiques, jusqu'au jour, récent, où émergea, sans grand succès à l'échelle planétaire, l'idée que le souverain serait soumis à un Etat de droit où il cesserait d'être le représentant incontrôlable d'une fonction magico-religieuse prétenduement garante de la prospérité générale - ou du destin de l'histoire.

Luc de Heusch

Université Libre de Bruxelles 


\section{Ouvrages cités}

Brown H.D.,

1944 "The Nkumu of the Tumba. Ritual chieftainship on the Middle Congo", Africa, XIV, 8, 431-447.

Clastres P.

1976 "Préface" in Sahlins M., Age de pierre, âge d'abondance. L'économie des sociétés primitives, Paris, Gallimard, 11-30.

Gilliard L.

1925 "Au roi Léopold II. Les Bolia. Mort et intronisation d'un grand chef", Congo, II, 223-239.

Heusch L, de,

1954 "Autorité et prestige dans la société tetela", Zaïre, X, 1001-1027.

1972 Le roi ivre ou l'origine de l'Etat, Paris, Gallimard.

Müller E. -W.

1955 Das Fürstentum bei den Südwest-Mongo (Belgisch Kongo), Dissertation doctorale inédite, Université de Mayence.

1957 "Le rôle social du 'nkumu' chez les Ekonda", Problèmes d'Afrique Centrale, VI, 281-289.

Stas J.B.,

1939 "Les nkumu chez les Ntomba de Bikoro", Aequatoria, 10-11, 109-123.

Sulzman E.

1959 "Die bikopo. Herrschaft der Bolia", Archiv für Rechts-und Sozial-philosophie, 55, 389-417.

Van der Kerken G.

1944 L'ethnie mongo, Bruxelles, I.R.C.B.

Van Everbroeck $\mathrm{N}$.

1961 Mbomb'Ipoku. Le seigneur de l'abîme, Annales du Musée de Tervuren.

Vansina J.

1964 Le royaume kuba, Tervuren.

1965 Introduction à l'ethnographie du Congo, Bruxelles/Kinsangani/Kinshasa/Lubumbashi.

1978 The children of Woot. A History of the Kuba Peoples, Madison, Wisconsin. 